\title{
Magnetic phase diagram of doped CMR manganites
}

\author{
Unjong Yu, Yookyung Jo, B. I. Min \\ Department of Physics, Pohang University of Science and Technology, Pohang 790-784, Korea
}

(November 20, 2018)

\begin{abstract}
The magnetic phase diagram of the colossal magnetoresistance (CMR) manganites is determined based on the Hamiltonian incorporating the double-exchange (DE) interaction between degenerate $\mathrm{Mn} e_{g}$ orbitals and the antiferromagnetic (AF) superexchange interaction between Mn $t_{2 g}$ spins. We have employed the rigorous quantum mechanical formalism and obtained the finite temperature phase diagram which describes well the commonly observed features in CMR manganites. We have also shown that the CE-type AF structure cannot be stabilized at $x=0.5$ in this model.
\end{abstract}

Keywords: CMR-manganites; Phase diagram; Double-exchange; Superexchange

Colossal magneto-resistance (CMR) manganites $\mathrm{R}_{1-x} \mathrm{~A}_{x} \mathrm{MnO}_{3}(\mathrm{R}=$ rare-earth; $\mathrm{A}=$ divalent cation $)$ have attracted much attention due to many unusual physical properties and complicated phase diagrams. [1]. The generic magnetic structure in the ground state is found to be $\mathrm{A} \rightarrow \mathrm{F} \rightarrow \mathrm{A} \rightarrow \mathrm{C} \rightarrow \mathrm{G}$-type with hole doping. $\mathrm{A}, \mathrm{C}$, and G-type denote layered type, rod type, and $\mathrm{NaCl}$ type antiferromagnetic (AF) structures, respectively. The major mechanism determining the magnetic structure of these compounds are the double-exchange (DE) which induces metallic ferromagnetism [2] and AF superexchange (SE) interactions.

The combined Hamiltonian of the DE and SE interactions is given by

$$
H=-\sum_{\langle i, j\rangle \alpha \beta} t_{0}^{\alpha \beta} \cos \left(\frac{\theta_{i j}}{2}\right) c_{i \alpha}^{\dagger} c_{j \beta}+J \sum_{\langle i, j\rangle} \mathbf{S}_{i} \cdot \mathbf{S}_{j} .
$$

Here, $t_{0}$ and $J$ are hopping and AF parameters, respectively. $\langle i, j\rangle$ denotes the nearest neighbor pairs and $\mathbf{S}_{i}$ is the local $t_{2 g}$ spin of the $i$ th site. $\alpha$ and $\beta$ represent the two orbitals of Mn $e_{g}$ band. $\theta_{i j}$ amounts to the distortion angle of spins at the sites $i$ and $j$. Brink and Khomskii [3] solved this Hamiltonian with the anisotropic mean field approximation in the classical limit. We have refined this approach; (i) by using the quantum mechanical treatment instead of taking the classical limit, and (ii) by generalizing the solution at the finite temperature.

The quantum mechanical expression of $\cos \left(\theta_{i j} / 2\right)$ is $\left(\hat{S}_{0}+1 / 2\right) /(2 S+1)$, where $S$ is the magnitude of local $t_{2 g}$ spin $(S=3 / 2)$, and $\hat{S}_{0}$ is the total spin of the sites $i$ and $j$ [4]. We adopted three order parameters, $\lambda, \Theta_{x y}$, and $\Theta_{z} . \lambda$ is the mean field determining whether the system is magnetically ordered or not, and $\Theta_{x y}$ and $\Theta_{z}$ are the average relative spin angles between neighboring sites in the $\mathrm{x}-\mathrm{y}$ plane and along the z-direction, respectively.

With these order parameters, the free energy per site can be comprised as follows.

$$
\begin{aligned}
& \frac{1}{N} F\left(\lambda, \Theta_{x y}, \Theta_{z}, T, y\right)= \\
& E_{\text {band }}\left(\lambda, \Theta_{x y}, \Theta_{z}, y\right)+E_{\mathrm{AF}}\left(\lambda, \Theta_{x y}, \Theta_{z}\right)
\end{aligned}
$$

$$
-T(y \sigma(\lambda, S=2)+(1-y) \sigma(\lambda, S=3 / 2)) .
$$

$E_{\text {band }}$ can be obtained by filling up the states with the electron number density $y(y=1-x) . \quad E_{\mathrm{AF}}$ denotes the SE energy and the entropy is given by $\sigma(\lambda, S)=$ $\log \nu-\lambda\left\langle S_{z}\right\rangle$, where $\nu$ is the partition function, and $\left\langle S_{z}\right\rangle$ is an expectation value of spin component in the direction of the mean field $\boldsymbol{\lambda}[5]$. By minimizing this free energy with respect to three parameters, one can determine the equilibrium phases. We have performed the rigorous quantum mechanical calculations for $\left(\hat{S}_{0}+1 / 2\right) /(2 S+1)$ and $\left\langle\mathbf{S}_{i} \cdot \mathbf{S}_{j}\right\rangle$.

The finite temperature phase diagram is given in Fig. 目 for $J / t_{0}=0.018$. At high temperature, the system becomes paramagnetic. At low temperature, the $\mathrm{G} \rightarrow \mathrm{C} \rightarrow \mathrm{A} \rightarrow \mathrm{F}$-type magnetic transitions appear with electron doping. $T_{\mathrm{N}}\left(T_{\mathrm{C}}\right)$ first decreases with electron doping and hits its minimum at the $\mathrm{C} \rightarrow \mathrm{A}$ phase transition point and then increases. This magnetic structure change and $T_{\mathrm{N}}$ minimum at $\mathrm{C} \rightarrow \mathrm{A}$ phase transition point coincide with the experimental observations in $\mathrm{Nd}_{1-x} \mathrm{Sr}_{x} \mathrm{MnO}_{3}$ [6]. Also notable is that the A-type $\mathrm{AF}$ ground state experiences a ferromagnetic transition in the doping region close to the ferromagnetic ground state before it becomes a paramagnetic state at high temperature. Indeed, this phenomenon is observed [7].

We have calculated the ground state energy of the CE-type [8] AF structure as a function of doping. Because the $\mathrm{C}$ and $\mathrm{CE}$-type have the same magnetic energy, the band energy alone determines the preferable ground state. From the inset of Fig. 1, one can verify that the CE-type has the lower band energy than the C-type for $y>0.54(x<0.46)$. Thus, it is impossible to explain the CE-type ground state near $x=0.5$ within this model. Prior to this result, Brink et al. [8] performed the same calculation in the classical limit. According to their result, the CE-type has the lower energy than the C-type for $x<0.57$ and they concluded that the CE-type naturally emerges near $x=0.5$ in the same model. Our results, however, indicates that their classical approximation fails at this point and that other mechanism such as charge, orbital ordering and structural distortion are 
required to explain the CE-type ground state. Finally, it should be pointed out that the description of the A-type AF near $x=0$ is beyond the scope of this model. In that case, the orbital degeneracy and the Jahn-Teller effect are thought to be crucial.

In conclusion, with the quantum mechanical calculation of the combined model of the DE and SE interactions, we have obtained the finite temperature phase diagram of doped perovskite manganites. We have also shown that the CE-type AF structure usually observed near $x=0.5$ cannot be explained within this model.

Acknowledgments- This work was supported by the KRF grant (KRF-2002-070-C00038).

[1] P. Schiffer, Phys. Rev. Lett. 75 (1995) 3336.

[2] C. Zener, Phys. Rev. 82 (1951) 403.

[3] J. van den Brink, D. Khomskii, Phys. Rev. Lett. 82 (1999) 1016.

[4] P.W. Anderson and H. Hasegawa, Phys. Rev. 100 (1955) 675.

[5] K. Kubo, N. Ohata, J. Phys. Soc. Jpn. 33 (1972) 21.

[6] Y. Tokura, Y. Tomioka, J. Magn. Magn. Mater. 200 (1999) 1.

[7] T. Akimoto, et al., Phys. Bev. B 57 (1998) R5594.

[8] J. van den Brink, et al., Phys. Rev. Lett. 83 (1999) 5118.

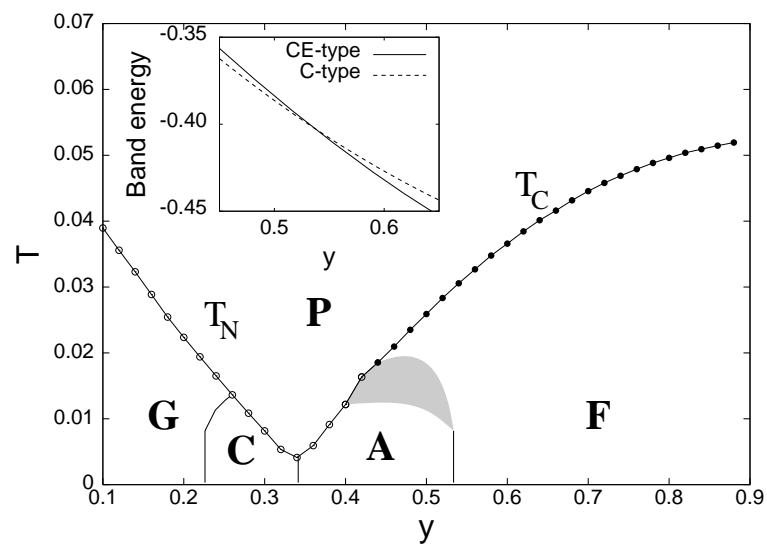

FIG. 1. Finite temperature phase diagram of doped manganites for $J / t_{0}=0.018$. The gray region represents a region where the stability between $\mathrm{A}$ and $\mathrm{F}$ states is indeterminable. (inset) Band energies of the $\mathrm{CE}$ and $\mathrm{C}$ types with respect to electron doping. 